\title{
Noise Elimination of Low-Voltage Power Line Communication Channel using Time-Frequency Peak Filtering Algorithm
}

\author{
Yuanju Mou ${ }^{1}$, Zhizhong $\mathrm{LV}^{2}$, Liang $\mathrm{GE}^{1}$, Xiaoting Xiao ${ }^{3}$, Zhengyin Wang ${ }^{1}$ \\ ${ }^{1}$ College of Mechanical and Electronic Engineering, Southwest Petroleum University, Chengdu, 610500, \\ China \\ ${ }^{2}$ Nanchong Key Laboratory Of Robotics Engineering And Intelligent Manufacturing, Southwest Petroleum \\ University, 63700, China \\ ${ }^{3}$ School of Electrical Engineering and Information, Southwest Petroleum University, 610500, China
}

Received: November 19, 2020. Revised: April 18, 2021. Accepted: May 7, 2021. Published: May 17, 2021.

\begin{abstract}
There are a lot of noises in the lowvoltage power line communication (LVPLC) channel, which seriously damages the LVPLC system. The noise in the low voltage power line can be divided into general background noise and random pulse noise. These two noises will cause serious interference to the communication process based on LVPLC, reduce the signal-to-noise ratio of LVPLC system, and the communication quality cannot meet the requirements. To ensure the communication quality, this paper uses the time-frequency peak filtering algorithm to eliminate the noise in the LVPLC in the experimental environment. Firstly, this paper studies the noise characteristics based on the measured LVPLC channel noise. Secondly, the memory noise model is established, and the time-frequency peak filtering algorithm is used to eliminate the noise. In order to analyze the denoising effect of time-frequency peak filtering algorithm, the algorithm is simulated. Finally, the application effect of the algorithm is verified by experimental test. The simulation and application results show that the timefrequency peak filtering algorithm can improve the signal-to-noise ratio by about $5 \mathrm{~dB}$ in the actual noise environment of LVPLC, which can adapt to the changeable environment of LVPLC channel noise, and has good noise suppression effect and good application
\end{abstract}

value. The application in the solar panel data transmission system shows that the time-frequency peak filtering algorithm can meet the communication performance requirements of the laboratory, reduce the bit error rate by about $2 \%$ under the general background noise interference, and reduce the bit error rate by about $3 \%$ under the pulse interference environment, and improve the transmission quality of LVPLC system.

Keywords - noise elimination; time-frequency peak filtering; noise model; finite memory; LVPLC.

\section{INTRODUCTION}

$\mathrm{L}$ ow-voltage power line communication(LVPLC) technology is a communication technology that relies on the power transmission line as the transmission medium to achieve data exchange [1]. It has the advantages of wide technical coverage, strong transmission capacity, low cost and high return. It is the key link to solve broadband transmission and realize the intervention of Ubiquitous Internet of Things, and has an irreplaceable position in the power grid construction[2]. In recent years, the LVPLC system has developed rapidly. Thirteen companies such as CISICO, Inter and HP in North America have established HomePlug Powerline Alliance, which mainly studies the field of home networking. Since 2001, 
HPA has released a series of communication standards such as HomePlug 1.0, HomePlug 1.0 Turbo, HomePlug AV and HomePlug AV2[3]. IEEE P1901 was established to promote the formulation of unified standards for broadband power line carrier communication technology. By studying the integration of HomPlug series standards and advanced data link control technology, IEEE 1901.2 standard was introduced to further improve the standard system[4]. Although the LVPLC system has developed rapidly and formulated a series of standards, how to use the low-voltage power line carrier communication technology to achieve reliable power line communication is still an important goal for technical personnel[5-6]. In order to improve the communication quality of low voltage power line communication system, scholars from various countries have studied the noise of low voltage power line channel[7]. At present, the research of low voltage power line communication channel noise is mainly concentrated in the following two aspects: Firstly, the noise characteristics of low voltage power line communication system are studied[8], and the influence of noise on power line communication system is studied by noise model[9]. research on noise elimination method of low voltage power line communication signal[10]. Although the study of LVPLC system noise is helpful to improve the quality of LVPLC, when the signal is submerged in a large number of noises, it will lead to low signal-to-noise ratio of LVPLC system quality of communication system[11-12]. Therefore, it is very important to study the noise elimination technology low SNR.

The SNR of LVPLC system is low, so noise cancellation and signal enhancement should be used in LVPLC system. In fact, in low voltage power line communication system, medium voltage power line communication system and high voltage power line communication system, the signal is always affected by pulse noise. Scholars in various countries have proposed various algorithms to suppress impulse noise, including algorithms based on error processing mechanism, methods based on time-frequency domain elimination and other types of noise algorithms[13] Forward error correction algorithm is the most common algorithm to suppress impulse noise based on error mechanism processing mechanism, which is widely used in power line communication as a channel coding technology, including Turbo coding system based on single carrier on the basis of statistical characteristics of impulse noise[14]. Using dual binary Turbo coding method [15-16], LDPC coding and decoding method [1718], etc., the main drawback of these noise suppression methods is that the complexity of coding and decoding is high, which will greatly increase the processing time of the system.In the traditional communication system, the early methods to reduce the impact of random impulse noise are parametric methods, such as assuming a specific statistical noise model and estimating the parameters of the statistical model at the training stage. Examples of this algorithm mainly include pre-filtering technology, blanking/clipping method, minimum mean square error symbol detector and iterative decoder[19]. The advantage of this parameterization method is that the system performance can be improved by using noise model and model parameters. However, such parties may lead to performance degradation of the whole system when noise models or parameters do not match. It is precisely because this method is excessively dependent on the parameter estimation of the system. Therefore, in subsequent studies, a large number of researchers have improved the accuracy of parameter estimation, such as some improved methods on the blanking/clipping method[20]. However, the limiting threshold is usually obtained by experiments and is not accurate enough. Although the improved method can improve the accuracy of the limiting threshold, the optimization of the threshold requires additional numerical software tools and cannot work well at low SNR[21-22]..

At present, the time-frequency peak filtering technology based on instantaneous frequency estimation is considered as the signal enhancement technology under low SNR conditions[23]. Time-frequency peak filtering algorithm is a signal enhancement method based on instantaneous frequency estimation[24]. The signal with noise is encoded as the instantaneous frequency of the frequency modulated analytic signal by frequency modulation. The peak value of the time-frequency distribution of the frequency 
modulated analytic signal is used as the instantaneous frequency estimation to eliminate noise by using the characteristics of its time-frequency distribution along the instantaneous frequency concentration[25-27].

This article takes the noise elimination problem in the solar panel data transmission system as the research background, proposes a time-frequency peak filtering algorithm to solve the noise in the solar panel data transmission system, and proves the feasibility of this method[24]. Through simulation and experimental algorithm. Through the test and analysis of the laboratory environment and solar panel data transmission system, the noise is eliminated by time-frequency peak filtering under the condition of low signal-to-noise ratio.

The rest of this article is arranged as follows. In Section 2, a time-frequency peak filtering algorithm for noise elimination is proposed.Section 3 verifies the effectiveness of the proposed algorithm by simulation. Section 4 is the experimental verification. The actual test and analysis are completed in the laboratory and the solar panel data transmission system. Section 5 is the conclusion and future direction.

\section{TIME-FREQUENCY PEAK FILTERING AlgORITHM}

\section{A. Instantaneous Frequency Estimation Method}

The time-frequency peak filtering technology is completed based on instantaneous frequency estimation. By frequency modulation, the noised signal is encoded as the instantaneous frequency of the analytic signal. The instantaneous frequency estimation is used to complete the estimation of the signal. The instantaneous frequency of the signal reflects the variation of the spectral peak position of the signal spectrum, and also the concentration of the signal energy on the time frequency plane [28]. Therefore, instantaneous frequency is an important parameter for studying non-stationary signals. It is also an important concept in time-frequency peak filtering [29].

A complex signal or an analytic signal $z(t)$ can always be expressed as $z(t)=a(t) e^{j 2 \pi \phi(t)}$, where $a(t)$ is the instantaneous amplitude, $\phi(t)$ is the instantaneous phase, and the instantaneous frequency of $z(t)$ can be defined as equation (1) [30]:

$f_{z}(t)=\frac{d \phi(t)}{d t}$

Therefore, on the other hand, signal $z(t)$ can be written as equation (2).

$z(t)=a(t) e^{j 2 \pi \int_{-\infty}^{t} f_{z}(\lambda) d \lambda}$

At present, the existing instantaneous frequency estimation methods are different for the different characteristics of instantaneous frequencies. These methods include direct method, zero-crossing detection method, phase modeling method and method based on the time-frequency distribution and so on. So far, the method based on time-frequency distribution has been mostly used for the instantaneous frequency estimation [31].

The estimation of the instantaneous frequency by the time-frequency distribution is obviously better than the non time-frequency one. On a time-frequency plane, the influence of the noise on the instantaneous frequency can be reduced by pre-processing, and the requirement for SNR can be lowered. Moreover, the multi-component signals can be separated on the time-frequency plane, thus the instantaneous frequency of each component can be estimated. For those signals with only frequency modulation as well as little amplitude modulation, especially for linear frequency-modulated (FM) signal, the signal energy of Vinag distribution is most concentrated along the instantaneous frequency. Hence, the instantaneous frequency of the signal can be obtained by the estimation of the Vinag distribution peak.

However, because the instantaneous frequency of the analytic signal is non-linear, instantaneous frequency estimation of Vinag's peak value will cause deviation. In this case, windowed Vinag, or called pseudo Vinag, can be used for frequency estimation. Pseudo Vinag distribution of the analytic signal (PWVD), is shown in equation (3) [32]: 
$W_{Z}(t, f)=\int_{-\infty}^{\infty} h(\tau) z^{*}\left(t-\frac{\tau}{2}\right)$

$z\left(t+\frac{\tau}{2}\right) e^{-j 2 \pi f \tau} d \tau$

In the above equation, $h(\tau)$ is the window function,

$W_{Z}$ is complex signal, $\tau$ is time constant.The instantaneous frequency estimation is the peak value of the frequency of pseudo Vinag distribution, as shown in equation (4).

$f(t)=\arg \max _{f}\left(W_{Z}(t, f)\right)$

\section{B. Time-Frequency Peak Filtering Noise Elimination Technology}

A real noisy signal processed by time-frequency peak filtering technology can be modeled as equation (5)[33]:

$$
s(t)=x(t)+n(t)
$$

where $x(t)$ is an effective signal that may consist of many band limited, non-stationary and deterministic components namely $x(t)=\sum_{k=1}^{p} x_{k}(t), n(t)$ represents additive white Gauss noise, and may overlap the spectrum with effective signal. The main objective is to restore the original signal $x(t)$ through the information contained in the noisy signal. However, it is difficult due to the overlapping of the noise and the signal spectrum. The time-frequency peak filtering method can restore the effective signal neatly without any hypothetical conditions. The specific steps of the time-frequency peak filtering are as follows:

Step1: Encode the noisy signal to make it an instantaneous frequency.

Perform frequency modulation to the noised signal $s(t)$, and obtain the analytic signal $z(t)$ of unit amplitude, as shown in equation (6)

$$
z(t)=e^{j 2 \pi \mu \int_{-\infty}^{t} s(\lambda) d \lambda}
$$

where $\mu$ is the frequency modulation index. According to the definition of instantaneous frequency; the noisy signal $s(t)$ is the instantaneous frequency of the analytic signal $z(t)$.

Step2: Obtain the peak value of the pseudo Vinag distribution of an analytic signal, that is, to estimate the instantaneous frequency of the analytic signal; The estimated value of the effective signal $x(t)$ is shown in equation (7).

$\hat{x}(t)=\hat{f}(t)=\frac{\arg \max \left(W_{z}(t, f)\right)}{\mu}$

Where $W_{z}(t, f)$ is the pseudo Vinag distribution of the analytic signal $z(t)$. For high noise cases, iterative time frequency peak filtering algorithm can be used, that is, to repeat steps (1) and (2) until it meets the requirement of the system.

\section{SIMULATION OF LVPLC SYSTEM}

The simulation of LVPLC system has been conducted to verify the effectiveness of the noise elimination algorithm. In the process, MATLAB was used to simulate the single carrier low voltage power line communication system, as shown in Fig.1. Thus, the effectiveness of the time frequency peak filtering algorithm can be analyzed. 


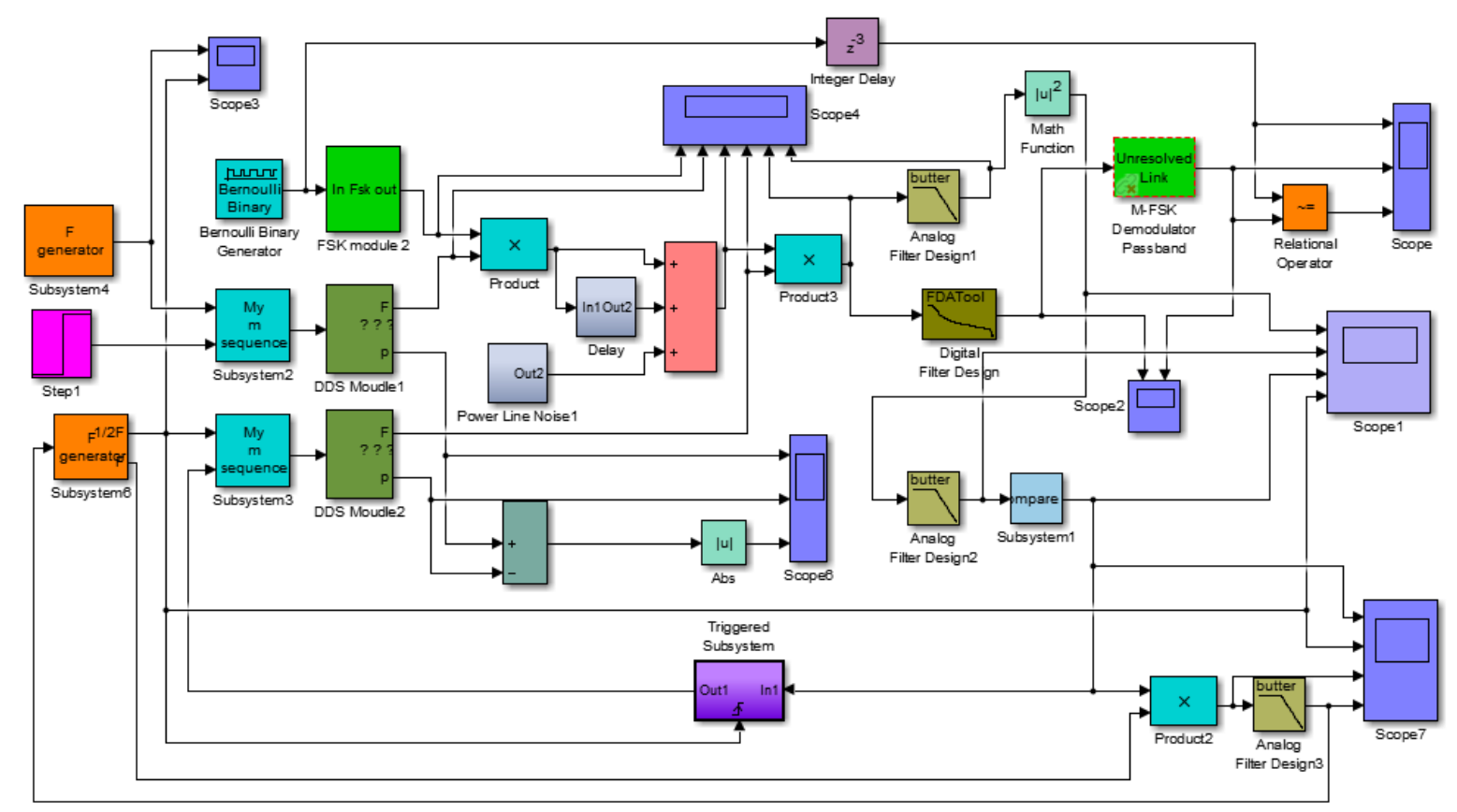

Fig.1 MATLAB noise modulation model

\section{A. The LVPLC System}

In the LVPLC system, many scholars have studied what kind of modulation technology is more suitable for LVPLC system. However, there is no uniform standard. Because the single-carrier system is simple in structure and low in cost, it is very suitable for low-rate transmission in the LVPLC system. Therefore, traditional frequency shift keying (FSK) and phase shift keying (PSK) have been studied and applied to the LVPLC system. Moreover, the multi-carrier system can effectively reduce multipath interference and reduce the influence of narrow-band interference and impulse noise. As shown in Fig.2, the single-carrier system can be used to effectively verify the effectiveness of the noise elimination technology.

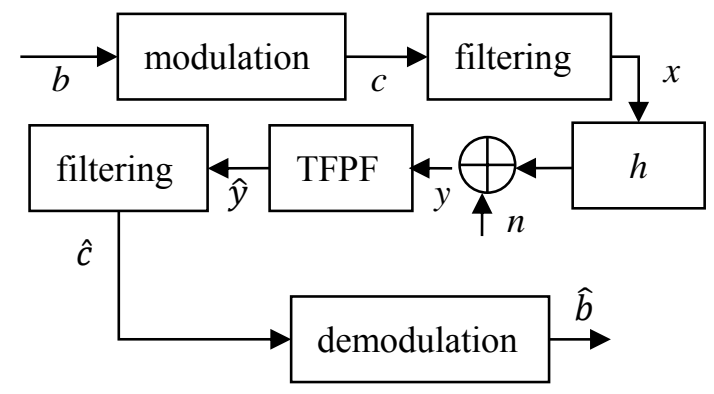

Fig.2 The LVPLC system for simulation
In the LVPLC system, due to the low transmitting power of the transmitter, even if the impulse noise is eliminated, the SNR may still be too low to ensure reliable communication. Therefore, only considering the background noise, The noise influence of the LVPLC system can be expressed as(8):

$y=x * h+n$

where $x$ is the pre-sent signal through the shaping filter, $y$ is the received signal through the LVPLC channel and noise impact, $n$ is the background noise of the LVPLC channel, $h$ is system function. For the received signal $y$, the time-frequency peak filtering algorithm is used to restore the original signal, in order to obtain the original signal and to eliminate the noise.

\section{B. Effectiveness of Time-Frequency Peak Filtering Algorithm}

Equation(9) is used to generate waveform , and then noise is added. Finally, the time-frequency peak filtering algorithm is used to denoise to verify the effectiveness of the algorithm. The signal generated by formula(9) is linear frequency modulationsignal $x(t)$, and the signal after adding noise is $y(t)$.The signal after denoising by time- 
frequency peak filtering algorithm is $y(t)$.

$$
x(t)=\cos \left(0.005 m+7.5 \times 10^{-7} m^{2}\right)
$$

To illustrate the effectiveness of the algorithm, first, compare the signal waveforms of $x(t), y(t)$ and $y(t)$, as is shown in Fig.3. Fig. 3 is drawn when SNR is $-4 d B$. From top to bottom, there are the waveforms of $x(t), y(t)$ and $y(t)$. It can be seen from the comparison of $x(t)$ and $y(t)$ waveforms that when $\mathrm{SNR}$ is $-4 \mathrm{~dB}$, the original waveform is seriously affected by noise, and the original waveform shape has been lost. Therefore, when LVPLC system has low SNR, the quality of communication will be greatly reduced due to the effect of noise. However, it can be seen from the comparison between the waveforms of $x(t)$ and $y(t)$ that their waveforms are very similar. Because of the time-frequency peak filtering algorithm, the noise is eliminated and the signal is restored to a certain extent.
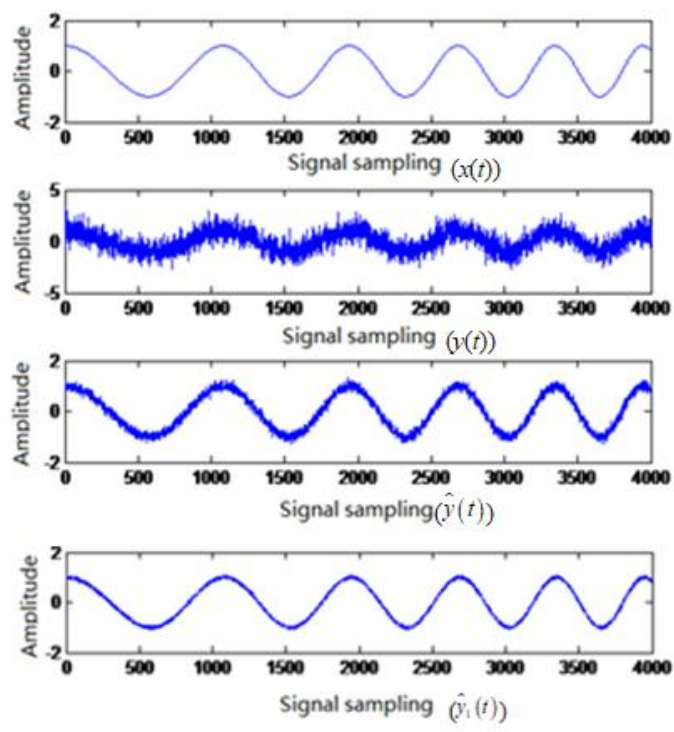

Fig.3 Waveform comparison of $x(t), y(t), \hat{y}(t)$ and $\hat{y}_{1}(t)$ Although the signal recovers to some extent, it can be seen from Fig. 3 that the signal does not reach ideal situation. The waveform still contains a lot of noise. In order to further remove the noise to improve the communication quality, the signal $y(t)$ can be processed with the time-frequency peak filtering again, and the results are recorded as $y_{1}(t)$. The comparison between the waveforms of $y(t)$ and $y_{1}(t)$ is shown in Fig.3. As we can see from Fig.3, the noise is clearly reduced after twice filtering, and the waveform is obviously similar to that of original signal. Therefore, in a very noisy environment, SNR cannot be raised to a satisfactory level only by filtering once. Thus, multiple iterations of time-frequency peak filtering algorithm can be performed to eliminate noise and improve the quality of signals, so as to achieve satisfactory results.

To illustrate the accuracy of the time-frequency peak filtering algorithm, the absolute errors((the difference between the measured signal and real signal) of the signals are compared, which can be seen in Fig.4. From top to bottom, they are respectively absolute errors between $x(t)$ and $y(t), x(t)$ and $y(t)$, and $x(t)$ and $y_{1}(t)$. It can be seen from Fig. 4 that due to the influence of noise, the absolute error between waveforms is large, which indirectly indicates that the signal is seriously disturbed. Because of the introduction of the time-frequency peak filtering algorithm, the absolute error is reduced, which indirectly shows that the signal waveform of the timefrequency peak filtering algorithm is closer to the original signal waveform, which also shows the effectiveness of the time-frequency peak filtering algorithm from another aspect. After the second time-frequency peak filtering algorithm, the absolute error is further reduced, and the signal is close to the original signal, which has very important guiding significance for signal recovery in low SNR and high noise environment. In the high noise environment, the iterative time-frequency peak filtering algorithm can be introduced into the communication system to reduce the noise influence and improve the communication quality greatly.

Through the above analysis, it can be concluded that the time-frequency peak filtering algorithm is effective for 
noise elimination in communication systems. If it is in a high noise environment, it can also reduce the influence of noise through multiple filtering.

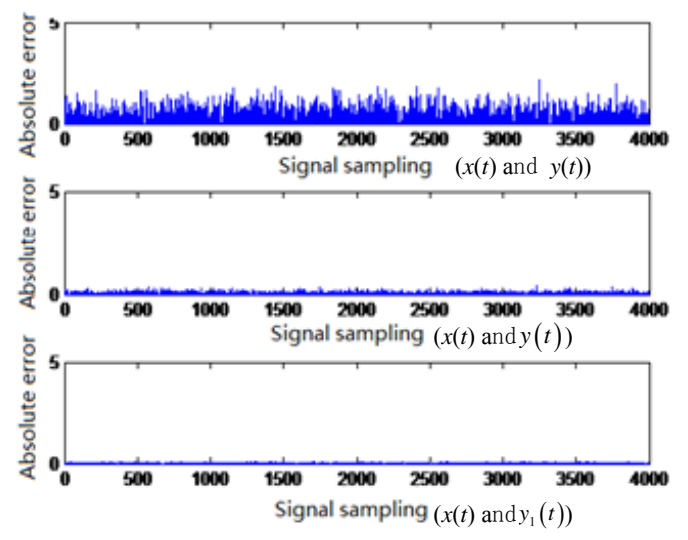

Fig.4 Absolute error comparisons

C. Verification of the Effectiveness of the Iterative Time-Frequency Peak Filtering Algorithm in

\section{LVPLC System}

Due to the existence of many noises and low emission power feature in commercial LVPLC systems, even in the case of impulse noise elimination, low SNR still exists in LVPLC system. The time-frequency peak filtering algorithm can effectively eliminate noise in low SNR situation. As has been proved above, multiple filtering, namely iterative time-frequency peak filtering algorithm can be applied to such noisy systems.

In order to illustrate the performance of the timefrequency peak filtering algorithm in LVPLC system, the Quadrature Phase Shift Keying(QPSK), Phase Shift Keying(PSK) and Frequency Shift Keying(FSK) communication systems are simulated, and the performances of Bit Error Rate(BER) are compared when SNR is $-10 \sim 0 \mathrm{~dB}$. Fig. 5 is the BER comparison diagram of the QPSK system, Fig.6 is the BER comparison diagram of the PSK system, and Fig.7 is the BER comparison diagram of the FSK system. In these figures, BER, not processed by the time-frequency peak filtering algorithm, is marked as "NO mitigation", and the BER is marked as "TFPF" after noise elimination by time-frequency peak filtering. The BER of PSK system is reduced by about $5 \%$,
FSK system is reduced by about $3 \%$, and QPSK system is reduced by about $2 \%$.

According to Fig.5-7, when there is no technology for noise elimination, the useful signals are submerged in the noise. This causes a very high bit error, lower quality of the communication system, as well as the insecurity and unreliability of the low voltage power line communication system. Therefore, to improve the quality of LVPLC system, the time-frequency peak filtering noise elimination algorithm is used to eliminate the influence of noise on the signal. From the figures, it can be seen that after using the time-frequency peak filtering algorithm, the BER of the system is greatly lowered, and the reliability is greatly improved. This indicates the effectiveness of the timefrequency peak filtering algorithm applied in the low voltage power line communication system.

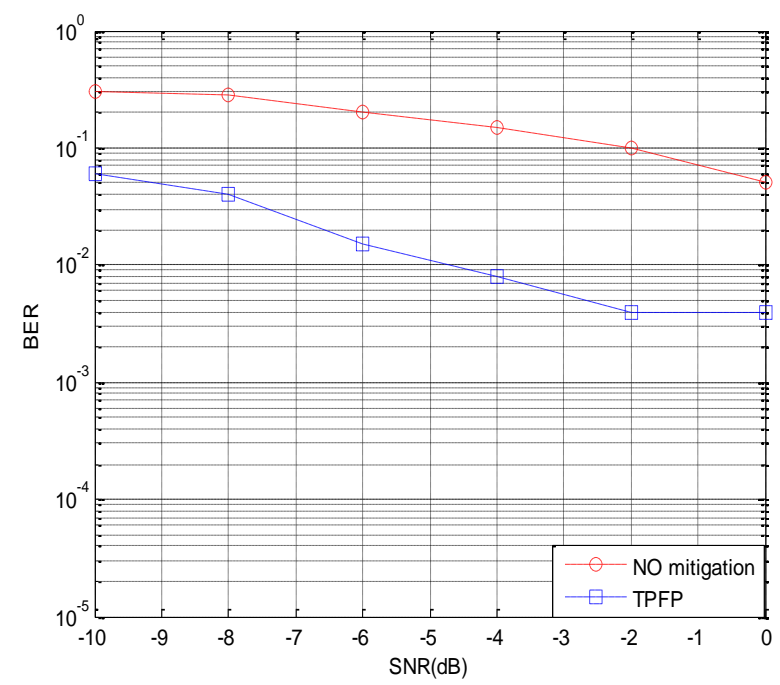

Fig.5 BER comparison diagram of PSK system 


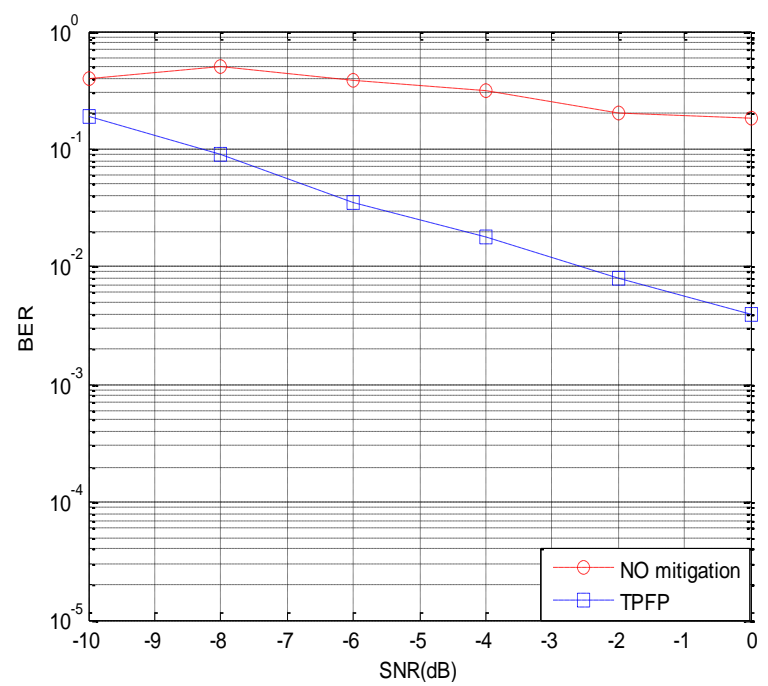

Fig.6 BER comparison diagram of FSK system

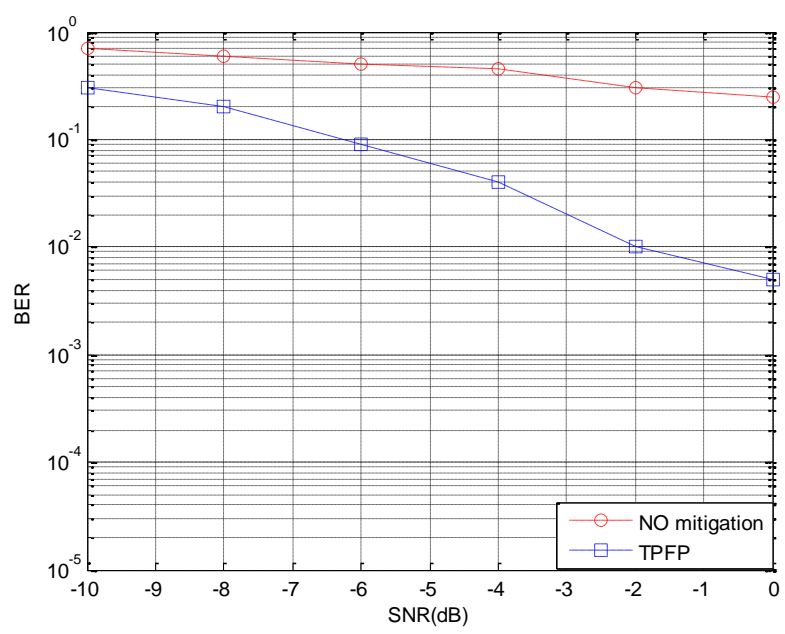

Fig.7 BER comparison diagram of QPSK system

\section{EXPERIMENTAL RESULTS AND ANALYSIS}

In order to verify the reliability of LVPLC system with noise elimination algorithm in the laboratory environment, the test of LVPLC channel with noise was carried out. The LVPLC carrier systems with interferences from different noises, such as colored background noise, narrow band noise, periodic impulse noise, synchronizing and asynchronizing with power frequency, and sudden impulse noise, were tested, respectively. This section selects representative test results and gives relevant analysis.

\section{A. Laboratory Test and Noise Elimination}

a. Introduction of laboratory noise sources

The principle of introducing relevant noise to household appliances is shown in Fig.8. The microprocessor engendered the signal with a certain high frequency, and these signals were coupled on the low voltage power line through the sending module and the coupling module. Then the corresponding noises was introduced in the low voltage power line though the house appliances. Finally, the signals were extracted through the coupling module and the receiving module, and transmitted to the upper computer interface for process. At the same time, the coupler also isolated the noise test device from the $50 \mathrm{~Hz}$ power frequency to ensure that the test equipment can work safely.

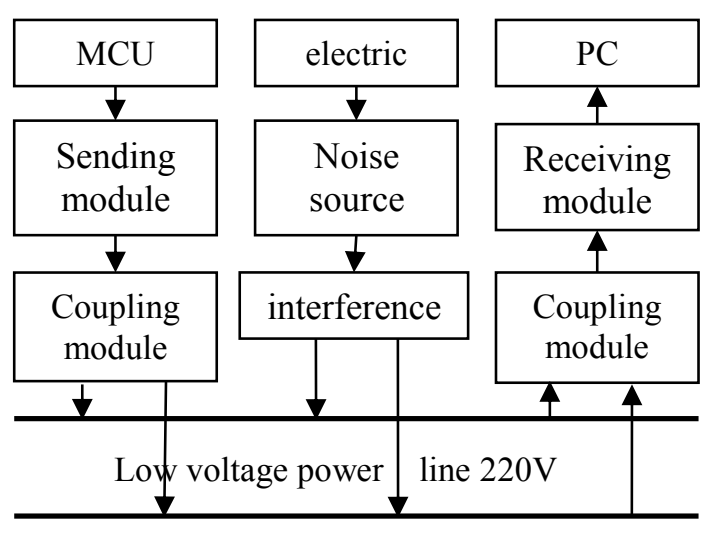

Fig. 8 the principle of noise introduction

b. Analysis of laboratory test results

Pure resistance electrical appliances are generally used for electric heat conversion, such as electric iron, electric heating rod and incandescent lamp. Such household appliances generally introduce colored background noise in LVPLC channel. Moreover, for the power load with an impulse width modulation transducer, the noise production mechanism and circuit structure had a close relationship. In the internal structure of this appliance, there was generally an impulse width modulation transducer. The function of this was to control the motor operation in insulated gate bipolar transistor driver. The operation of the machine would introduce the periodic impulse noises synchronizing with the power frequency into the low voltage power line communication channel. The periodic impulse noise with regularity and certain intensity, which was asynchronous with power frequency, was introduced into the low voltage power line every half power frequency cycle of the rectifier. And periodic pulse noise source asychronizing with power frequency would be introduced generally into LVPLC for electrical appliances or devices 
that contained rectifier circuits, such as mobile phone chargers, computer adapters and LED lights. The correct reception data rate without using the time-frequency peak filtering technology was marked as "NO mitigation", and the correct reception data rate after the noise elimination by time-frequency peak filtering was marked as "TFPF". The experimental results are shown in Figure 9.

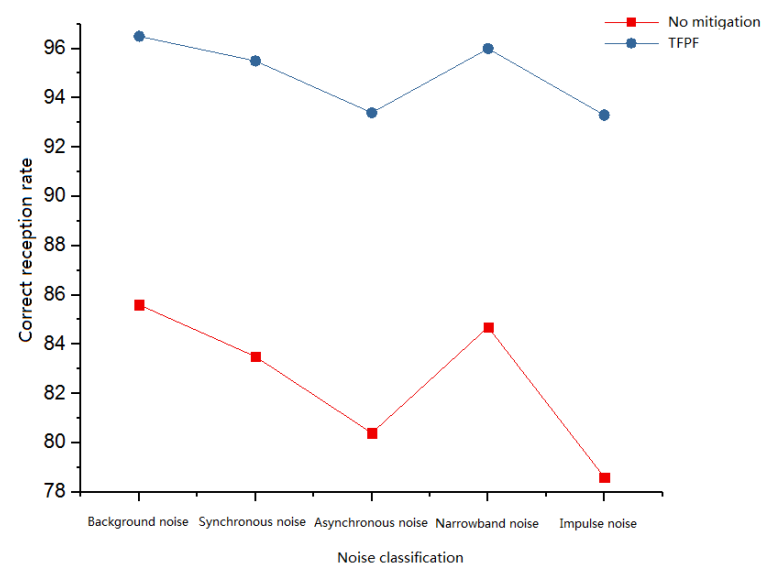

Fig.9 Experimental test results

According to Fig.9, the correct receiving data rate can be improved to about $95 \%$, after being processed by the time frequency peak filtering noise elimination algorithm. The communication quality of the LVPLC system can be improved effectively under any noised environment.

B. Test in the Data Transmission System of the Solar Panel

a. Experimental platform

The principle of applying the LVPLC system into the data transmission system of the solar panel is shown in Fig.10. The data transmission line of the solar panel was not needed when applying the low voltage power line communication system into the automatic detection of the solar panel transmission. The low voltage power line carrier technology can be applied, with the solar energy output circuit as the signal transmission media, to carry out real time transmission of the solar panel data.

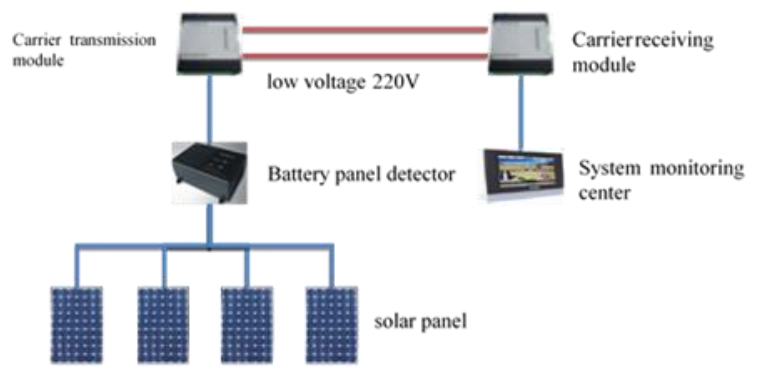

Fig.10 Test platform

The information of the voltage and temperature was transmitted through the LVPLC, with the receiving module of the LVPLC carrier connected to the monitoring host computer and the sending module connected to the collection equipment. Due to the limitation of the experimental conditions, communication applications with a maximum length of around 200 meters were carried out. The pollution of the LVPLC carrier channel was relatively light, and the testing environment were similar to some extent, comparing the relatively pure environment with a small number and single type of appliance and stable appliance switches, such as the solar panel. The system software is installed on the host computer. The working states of al solar panels can be observed through the monitoring software, and the collected data of voltage and temperature were displayed in real time. Through the collected information by the monitoring software, the system host could judge whether the solar panel was damaged. When the voltage was lower than the set alarming value, or the temperature of the solar system was higher than the environment in normal working, the system would report the fault point automatically, and show the position of the fault. Thus, it is convenient for the maintainers to reach the fault area and treat the faulted solar panel.

b. System application analysis

The LVPLC transmission module in the data transmission system controlling platform of the solar panel in Southwest Petroleum University is single-phased intelligent meter carrier module (TXZX13-MR21M). Through the comparison with the power line carrier module studied in this paper, the adaptation of the LVPLC carrier module can be instructed. The comparison of the two modules is shown in table 1 . 
The experimental data with $9600 \mathrm{bit} / \mathrm{s}$ baud rate and 200meters communication distance was selected. Then all kinds of performances of the modules both in the paper and product were compared, and the curves in Fig.11-14 can be obtained.

\begin{tabular}{|c|c|c|c|c|c|c|c|}
\hline Hardware & $\begin{array}{l}\text { Temperatur } \\
\mathrm{e}\end{array}$ & Voltage & Sensitivity & $\begin{array}{c}\text { Grid } \\
\text { environment }\end{array}$ & $\begin{array}{l}\text { Isolation } \\
\text { voltage }\end{array}$ & $\begin{array}{c}\text { Communication } \\
\text { rate }\end{array}$ & $\begin{array}{l}\text { Carrier } \\
\text { frequency }\end{array}$ \\
\hline Thesis module & $-40-85^{\circ} \mathrm{C}$ & $5-20 \mathrm{~V}$ & $20 \mathrm{mV}$ & $\mathrm{AC}<305 \mathrm{~V}$ & $4 \mathrm{kV}$ & $6.7 \mathrm{Kbit} / \mathrm{s}$ & $72 \mathrm{~K}$ \\
\hline Product module & $-40-85^{\circ} \mathrm{C}$ & $12-15 \mathrm{~V}$ & $30 \mathrm{mV}$ & $\mathrm{AC}<305 \mathrm{~V}$ & $4 \mathrm{kV}$ & $1.6 \mathrm{Kbit} / \mathrm{s}$ & $76.8 \mathrm{~K}$ \\
\hline
\end{tabular}

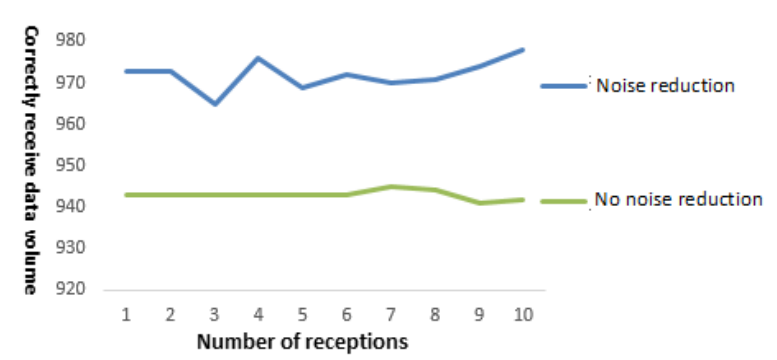

Fig.11 Narrow band noise interference

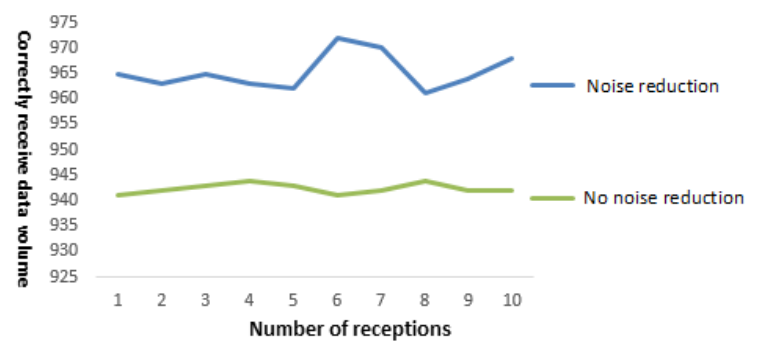

Fig.12 Colored background noise interference

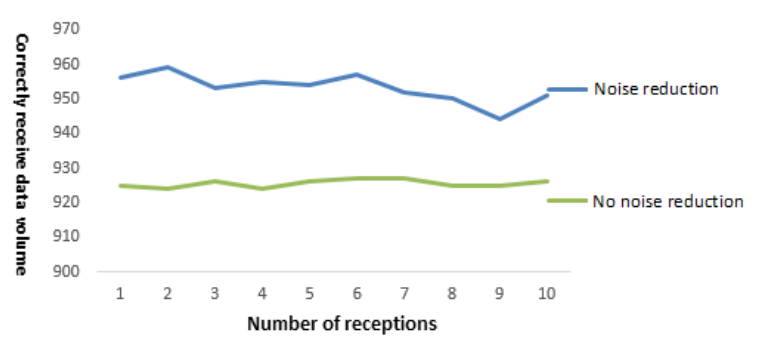

Fig.13 Asynchronous periodic impulse noise interference

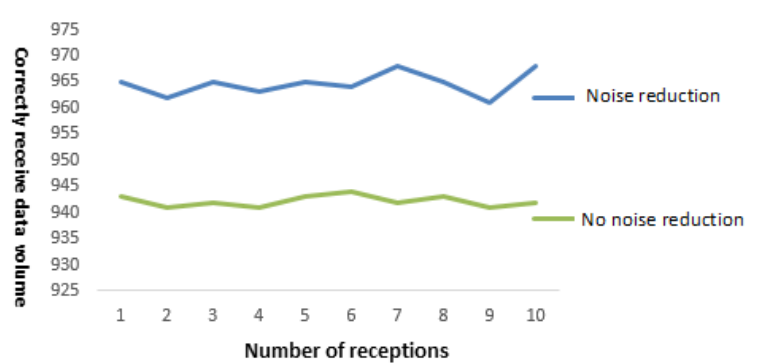

Fig.14 Synchronous periodic noise interference

be seen that the low voltage power line carrier module used

From Figure 12, Figure 13, Figure 14, Figure 15, it can in this paper under the interference test of $200 \mathrm{~m}$ at 9600 
baud rate, in the case of the same number of reception, the correct data received are greatly increased. It can be seen from Fig. 12, Fig. 13 and Fig. 15 that the error rate of the low-voltage power line carrier module is approximately $2 \%$ lower than that of the single-phase smart meter product module in the console under the general background noise interference It can be seen from Figure 14 that the bit error rate of the low voltage power line carrier module is about $3 \%$ lower than that of the single-phase smart meter product module in the console in the pulse interference environment, and the signal-to-noise ratio is increased by about $5 \mathrm{~dB}$ in the actual low voltage power line communication noise environment. It shows that the timefrequency peak filtering algorithm used in low voltage power line carrier module in this paper has the best effect on noise elimination in low voltage power line carrier products under the pulse interference environment of 200 $m$ at 9600 baud rate, and also shows the applicability and effectiveness of the time-frequency peak filtering algorithm in low voltage power line carrier products. It can be seen from table 1 that the working voltage of the LVPLC module (5-20V) is lower than that of the singlephase smart meter product in the console $(12-15 \mathrm{~V})$, and the lower working voltage means that the power consumption of the system is lower. That is to say, the LVPLC module is more suitable for low-voltage power line carrier communication. The static power consumption of the low voltage power line communication module at $0.25 \mathrm{~W}$ is lower than that of the single-phase smart meter product in the console at $0.35 \mathrm{~W}$. The rate of $6.7 \mathrm{Kbit} / \mathrm{s}$ of the low voltage power line communication module is higher than that of the single-phase smart meter product module in the console at $1.66 .7 \mathrm{Kbit} / \mathrm{s}$. In terms of carrier frequency, the LVPLC module is set to $72 \mathrm{~K}$, which is lower than the carrier frequency of $76.8 \mathrm{~K}$ of single-phase smart meter products. The higher the carrier frequency is, the smaller the signal attenuation is. However, the load change of lowvoltage power line is frequent, and the power consumption will increase. From the perspective of power consumption and cost, this carrier frequency is more suitable for lowvoltage power line carrier communication.

The current transmission of solar panel data contains temperature, current, voltage three kinds of data, each data using a separate packet to send, the packet contains boot code, packet information, CRC check code and user data with a total length of $80 \mathrm{bit}$, the three kinds of data of a solar panel need three packet total length of 240 bit. A solar panel group has 4 solar panels, and the data are updated every 1 minute. Using 1600 bps transmission speed, the data can be transmitted for 100 times $(1600 \mathrm{~b} / \mathrm{s} \times 60 \mathrm{~s} \div 240 \mathrm{bit} \div 4=100)$ repeatedly in the required updating time. Thus, time-frequency peak filtering algorithm can reduce the bit error rate of low voltage power communication system and improve the communication quality of the system. From the perspective of cost and power consumption, the low voltage power line carrier module developed in this paper greatly reduces the cost and power consumption, which is of great significance to practical engineering and can fully meet the communication requirements of practical engineering.

\section{CONCLUSION}

This paper conducts in-depth research on the timefrequency peak filter algorithm, completes the simulation design of the time-frequency peak filter algorithm, and studies the method of noise elimination for LVPLC signals. After testing and analyzing the laboratory environment and the solar panel data transmission system, In the case of low signal-to-noise ratio, use time-frequency peak filtering to eliminate noise.

Through testing and analysis, the following conclusions are obtained:

1) In view of the low signal-to-noise ratio, the timefrequency peak filtering algorithm is chosen as the noise elimination technology for LVPLC systems. The simulation results show that the time-frequency peak filter algorithm can effectively suppress noise.

2) According to the time-frequency peak filter algorithm simulation results, the laboratory introduces interference source testing. The experimental test results show that the correct received data rate is increased to $95 \%$ after the time-frequency peak filter noise elimination algorithm, which improves the 
SNR ratio of the LVPLC system.

3) The LVPLC system with time-frequency peak filtering denoising function is applied to the data transmission system of solar panels. The application results show that the LVPLC carrier module is approximately $2 \%$ lower than the single-phase smart meter product module in the console under the general background noise interference, and the bit error rate is reduced to about $3 \%$ in the pulse interference environment. In the actual LVPLC noise environment, the signal-to-noise ratio is increased by about $5 \mathrm{~dB}$, indicating the applicability and effectiveness of the time-frequency peak filtering algorithm in LVPLC carrier products.

With the development of smart grid and the continuous expansion of power line communication business and service scope, more and more requirements will be put forward for the service of high-speed communication in low-voltage power grid. At the same time, the safety and quality of communication will also be higher and higher. Therefore, the study of better and faster noise cancellation technology to ensure the quality of low voltage power line communication, in order to better serve different types of power line communication business, will be the focus of high-speed communication in low voltage power grid. This paper only studies the asynchronous impulse noise elimination algorithm in low voltage power line communication system, without considering the influence of cyclic impulse noise in low voltage power line communication system, and without considering the environment of multiple input and multiple output. Therefore, in the future, the noise elimination technology under cyclic impact noise environment can be considered, including the modeling and simulation of cyclic impact noise, the research and simulation of noise elimination algorithm. The noise elimination technology in multi-input multi-output system is studied. Considering the complexity of the existing algorithm, the superiority of the algorithm is maintained and the complexity of the algorithm is reduced.

\section{ACKNOWLEDGMENTS}

This work is supported by the National Natural Science Foundation (51974273), the International Science and Technology Cooperation and Exchange Research Project of Sichuan Province (18GJHZ0195), Downhole Intelligent Measurement and Control Science and Technology Innovation Team of Southwest Petroleum University (2018CXTD04), Fund of National Engineering and Research Center for Mountainous Highways ( GSGZJ2020-01) and the international Science and Technology Cooperation Project of Chengdu(2020-GH02-00016-HZ).

\section{References}

[1] Cano C, Pittolo A,Malone D.State-of-the-art in power line communications:from the applications to themedium[J].IEEE Journal on Selected Areas in Communications,2016,34(7):19335-1952.

[2] Guo Yihe.,Huo Ran., Liu Xin., Xie Zhiyuan., Qiu Juan.,Lumped Parameter Cable Model for Low Voltage Power Line Communication[J].Dianli Xitong Zidonghua/Automation of Electric Power Systems, 2019, 43(02): 156-161.

[3] Khalil K, Gazalet M G, Corlay P.An MIMO ran dom channel generator for indoor power-line com munication[J].IEEE Transactionson Power Deliver y,2014,29(4):1561-1568.

[4] Develi I, Kabalci Y, Basturk A. Artificial bee colony optimization for modelling of indoor PLC channels: A case study from Turkey[J].Electric Power Systems Research,2015,127(3):73-79.

[5] Cui Ying., Liu Xiaosheng., Xu Dianguo., Low-voltage Power Line Communication[J]. Dianli Xitong Zidonghua/Automation of Electric Power Systems, 2019, 43(24): 111-118

[6] Van Rensburg, Petrus A.Janse., Snyders, Abraham J., Ferreira, Hendrik. Modeling of Coupling Diversity for Extra-Low-Voltage Power-Line Communication [J].IEEE Journal of Emerging and Selected Topics in Power Electronics, 2018, 6(3):1224-1234.

[7] Liu Xiaosheng,Cui Ying,Xu Dianguo.Performance optimization for low voltage power line 
communication[J].IEEE Transactions on Power Delivery, 2017, 37(12): 16-21 and 24.

[8] Francesco Chiti, Romano Fantacci, Andrea Tani. An efficient network coding scheme for reliable multicast power line communications[J]. Physical Communication, 2017. 35: 78-85.

[9] Zhang L, Liu X.S, Pang J.W,Xu D.G,Leung V.C.M . Reliability and survivability analysis of artificial cobweb network model used in the low-voltage power-line communication system $[\mathrm{J}]$. IEEE Transactions on Power Delivery, 2016, 31(05): 1980-1988.

[10] Zhang Liang, Liu Xiaosheng, Qi Jiajin,Zhou Ya $\mathrm{n}, \mathrm{Xu}$ Dianguo . Study of improved hierarchica 1 ant colony routing algorithm for low-voltage $p$ ower line communication $[\mathrm{J}]$. Diangong Jishu $\mathrm{X}$ uebao/Transactions of China Electrotechnical Soc iety, 2014, 29(02): 318-324.

[11] Xiang Min, Wen Chao, Hou Xing-Zhe. A hybrid relay control mechanism for ribbon topology in lowvoltage power line communication networks $[\mathrm{J}]$. International Journal of Communication Systems, 2015, 28(03): 585-600.

[12]Francesco Chiti, Romano Fantacci, Andrea Tani. An efficient network coding scheme for reliable multicast power line communications[J]. Physical Communication, 2017. 35: 78-85.

[13] Peter A O.Power line communication(PLC) impulsive noise mitigation:a review[J].Journal of Information Engineering \& Applications,2014.

[14]Tseng D,Tsai T,Han Y S.Robust turbo decoding in impulse noise channels[C].In:IEEE International Symposium on Power Line Communications and ITS Applications.Univ Johannesburg,Sch Tourism \& Hospital,Johannesburg,SOUTH

AFRICA:IEEE,2013,230-235.

[15]Kim E C,I S S,Heo.Performance of double binar yturbo coding for high speed PLC systems[J].IEE E Transactions on Consumer Electronics,2010,56( 3):1211-1217.

[16]Himeur Y, Boukabou A.OFDM-based power-line comminication enhancement using a turbo coded adaptive impulsive noise compensator[J].Telecom munication,2016,62(3):481-94.

[17]Lin J, Nassar M, Evans B L. Impulsive Noise Mitigation in Power line Communications Using Sparse Bayesian Learning[J]. IEEE Journal on Se lected Areas in Communications, 2013, 31(7):117 2-1183.

[18] Liu Shugang, Zhang Hongtai, Liu Hongli. Decoding optimization method for BP in low-voltage powerline communication $[\mathrm{J}]$. Dianwang Jishu/Power System Technology, 2014,38(10): 2828-2832.

[19] Toshiyuki Igarashi, Koichi Kondo, Shigeyoshi Yoshida. Design and evaluation of noise suppression sheet for $\mathrm{GHz}$ band utilizing magneto-elastic effect[J]. Journal of Magnetism and Magnetic Materials, 2017, 23: 444-458.

[20] Souma Jinno, Hiroshi Toki, Masayuki Abe. Mechanism of common-mode noise and heat generation in an electric circuit with grounding using multiconductor transmission-line theory $[\mathrm{J}]$. Chinese Journal of Physics, 2017, 55(3): 145-159.

[21]Bin Han, Vlad Stoica, Cornelius Kaiser, Nico Otterbach, Klaus Dostert. Noise characterization and emulation for low-voltage power line channels across narrowband and broadband $[\mathrm{J}]$. Digital Signal Processing, 2017, 69: 189-197

[22]Lucile Rutkowski, Piotr Masłowski, Alexandra C. Johansson, Amir Khodabakhsh, Aleksandra Foltynowicz. Optical frequency comb Fourier transform spectroscopy with sub-nominal resolution and precision beyond the Voigt profile[J]. Journal of Quantitative Spectroscopy and Radiative Transfer, 2018, 33: 204-219.

[23] Ke Jia, Chenjie Gu, Lun Li, Zhengwen Xuan, Tianshu Bi, David Thomas. Sparse voltage amplitude measurement based fault location in large-scale photovoltaic power plants[J]. Applied Energy, 2018, 45: 211-223.

[24] GUZEL T,USTUNEL E,CELEBI H,et al. Noise modeling and OFDM receiver design in power-line communication[J].IEEE Transaction on Power Delivery,2011,26(4):2735-2742. 
[25]BOASHASH B, MESBAH M. Signal enhancement by time-frequency peak filtering $[\mathrm{J}]$. IEEE Transactions on Signal Processing, 2004,52(4):929937.

[26] TIAN Y, LI Y. Parabolic-trace time-frequency peak filtering for seismic random noise attenuation[J]. IEEE Geoscience and Remote Sensing Letters, 2014,11(1):337-341.

[27] Guiru Liu, Mingzheng Zhou, Lulin Wang, Hai Wang, Xiansheng Guo. A blind spot detection and warning system based on millimeter wave radar for driver assistance[J]. Optik - International Journal for Light and Electron Optics, 2017, 135: 156-169.

[28] Liu Yanping, Yan Zhengguo. Application of a cascading filter implemented using morphological filtering and time-frequency peak filtering for seismic signal enhancement [J]. Geophysical Prospecting, 2020, 68(06): 1727-1741.

[29]Zhao X, Li Y , Zhuang G, et al. 2-D TFPF based on Contourlet transform for seismic random noise
attenuation[J]. Journal of Applied Geophysics, 2016, 129:158-166.

[30] Liu Naihao, Yang Yang, Li Zhen, Gao Jinghuai,Jiang Xiudi, Pan, Shulin. Seismic signal de-noising using time-frequency peak filtering based on empirical wavelet transform [J]. Acta Geophysica, 2020, 68(02):425-434.

[31] pursuit algorithm and power line noise suppression of magnetotelluric signal[J]. IOP conference Series: Earth and Environmental Science, 2017,94(1):78-83.

[32] Ren, Huorong; Ren, An; Li, Zhiwu. A new strategy for the suppression of cross-terms in pseudo WignerVille distribution[J]. Signal Image and Video Processing.2016,10(1):139-144.

[33] SU Lingdong,ZHAI Mingyue,HE Xin, A new noise mitigation method based on time frequency peak filtering in powerline communication system $[\mathrm{J}]$. Power System Protection and Control.2015,43(1):1520.

\section{Creative Commons Attribution License 4.0} (Attribution 4.0 International, CC BY 4.0)

This article is published under the terms of the Creative Commons Attribution License 4.0

https://creativecommons.org/licenses/by/4.0/deed.en_US 